\title{
Damage detection and localization in composite beam structures based on vibration analysis
}

\author{
Samir Khatir*, Idir Belaidi*, Roger Serra**, Magd Abdel Wahab***, Tawfiq Khatir**** \\ *Department Of mechanical engineering University M'hamed Bougara Boumerdes, LEMI Laboratory Research Team Modelling \\ and Simulation in Mechanical Engineering, 35000 Boumerdes, Algeria, \\ E-mail: Khatir_samir@hotmail.fr,idir.belaidi@gmail.com \\ **Laboratoire de Mécanique et Rhéologie INSA Centre Val de Loire, LMR, 3 Rue de la chocolaterie, 41000 Blois, France, \\ E-mail: roger.serra@insa-cvl.fr \\ ***Applied Mechanics Laboratory Soete Faculty of Engineering and Architecture Ghent University \\ Technologiepark Zwijnaarde 903B-9052 Zwijnaarde, Belgium E-mail: Magd.AbdelWahab@UGent.be \\ ****Institute of science and technology University Centre Salhi Ahmed, Naama 45000, Algeria, \\ E-mail: khatir-usto@hotmail.fr
}

cross $^{\text {ref }}$ http://dx.doi.org/10.5755/j01.mech.21.6.12526

\section{Introduction}

Composite materials are nowadays increasingly used as an alternative to conventional materials, because of their high strength, weight saving, specific rigidity, and mechanical flexibility especially in the aerospace industry. The aim of vibration based damage detection techniques is to determine the occurrence of structural damage, its location and severity. The information produced by a damage assessment process can play a vital role in the development of economical repair and retrofit program. Rytter [1] proposed a classification in order to allow a comparison between different techniques, which consists of four levels. The first level is the detection, the second level is the localization, the third level is the assessment, and finally the fourth level, which is the consequence of damage, predicts the remaining life and the actual safety of the structure in a certain state of damage. The complete health state of a structure can be determined based on presence, location, type and severity of damage (diagnostics) and estimation of remaining useful life (prognostics) [2]. The concept of dynamical invariants [3] in the SHM methodology is called Beacon-based Exception Analysis for Multi-missions (BEAM).

Methods of identification of defects and their analysis in a qualitative relation to the location of defect and its importance have been studied in literature [4-5]. Noise does not affect stable low-order dynamical models that can be created using POD based low-order model for fault detection [6].

POD provides the most efficient way of capturing the dominant components of an infinite-dimensional process with only (often surprisingly) few modes. Various applications of POD to structural dynamics were carried out in the literature [7-11]. The diagnostics of various machines and mechanisms is an important problem to determine the damaged structural elements. Solving of such problem, for example use the method of resonance frequencies, the damaged structural element is judged by the deviation of resonance frequency [12]. The location of damage in the structure is more complicated for certain class of structure, e.g. a beam-like structure, using vibration analysis. In this approach, the beam structure is successively loaded with mass. A harmonic force is used to excite the structure in the loaded zones. The detection and localization of damage are indicated by the relationship between vibration amplitudes of the additional mass and its location [13]. Damage detection of a bridge structure based on computer simulations of static displacement or strain data using POD [14] and finite element analysis had shown a success detection. The damage indicator based on mode shape data was introduced [15] to identify damage in beam-like structures. A two-step procedure for damage detection in structures from changes in curvature mode shapes was proposed [16]. The damage identification and localization of some complex mechanical system described in terms of reduced number of modes using finite elements was reported [17], where an isolation procedure to describe these parameters was followed. Mathematical simulations of structural elements and dynamic behaviour due to loss of stiffness at damaged area were presented [18].

The most existing damage detection study was based on modal curvature and investigated the indicator value changes between the intact and damaged states [19]. The processing of nonlinear features associated with a damage event by quadratic time frequency distributions for damage identification in a frame structure were studied [20].

A simple method for determining the stiffness matrix of structural and mechanical systems using measured natural frequencies and corresponding mode shapes was proposed [21]. The use of natural frequency shifts for damage identification was proposed in several research works, where the success of this parameter in the case of a single damage location was proven [22]. The first four natural frequencies of a simulated cantilever beam were used to locate a single crack [23]. The identification of a single crack in an experimental single story frame from shifts in the first three natural frequencies was investigated [24] using a damage identification algorithm to locate and identify the size of a single crack. The identification of a single crack in a vibrating rod based on knowledge of the damage induced shifts of a pair of natural frequencies was investigated [25]. A damage identification methodology based on natural frequency changes to a numerical model of a beam on an elastic foundation was studied [26].

Current electrodynamic vibrators and vibration rigs for monitoring materials, structural elements and ma- 
chine parts objects subjected to vibrations and large accelerations were used to detect damage [27]. The thrusting force and amplitude of oscillations in electrodynamic vibrators are discussed along with broadening of the frequency range.

The results found in reference [28] identified single damage events as stiffness loss, connection loosening and lump mass addition. Two methods of damage assessment based on a relationship between modal strain energy and measured modal properties tailored to single damage cases [29] were used. Damage approach prediction in beam and plate structures with initiated damage were presented [30]. The results provided the basis for the development of diagnostics algorithms. The location and severity of a single damaged element in a simulated planar truss were determined by minimizing the square of the Residual Force Vector (RFV) [31].

In the present work, a new damage identification method is applied to a composite beam structure using genetic algorithm and particle swarm optimization. By introducing the proper orthogonal decomposition with radial basis function, a reduced model is built, the calculation of cost function is minimized and more accurate results are obtained.

A finite element model of bi-dimensional monolithic beam reinforced by a graphite-epoxy discretized into 10 elements is used to generate vibration data. The damage resulted in reduction of stiffness with levels of $5 \%$ and $25 \%$, which are placed in different positions. A comparative study between both results of (GA) and (PSO) using finite element method indicates that PSO is better than GA. However, the calculation of PSO takes a longer time, with small error between the real and estimated damage. The PSO with POD is better than PSO with FEM algorithm to detect and localize damage with higher accuracy and shorter computational time. The effect of noise in this methodology is considered in some cases by assigning noises to natural frequencies.

\section{Theoretical background}

\subsection{Proper orthogonal decomposition (POD) with radial} basis functions (RBF)

POD with Radial Basis Functions (RBF) is used for the interpolation of the data with previously reduced dimensionality by the POD. A group of responses for a given system can be very effectively compressed using the theory of a separate POD. This compression allows for a significant reduction of the dimensionality. To make it clear, let us recall that our goal is to define an approximation that should be used instead of FE simulations. Therefore, we wish to find a function that depends on some parameters collected in vector $p$ such that:

$$
f(p)=u \text {. }
$$

In Eq. (1), vector $\mathrm{u}$ collects the required output of the system and represents the frequency vector of a damaged beam modelled using FEA.

However, since we already constructed a low order approximation of these responses, they can be represented in the new truncated system by the matrix of amplitudes. This practically means that RBF can be applied in already reduced dimensionality, where responses are expressed as amplitudes, and therefore the function we are looking for is in the following form:

$$
f a(p)=\bar{a} \text {. }
$$

The relationship previously defined between the responses expressed in the reduced and full dimensionality holds for the functions $f$ and $f a$. Thus we can write:

$$
f(p)=\bar{\Phi} f a(p)=u
$$

Applying the Radial Basis Functions (RBF) technique, the approximation of $f a$ is written as a linear combination of some basis functions $g_{i}$, i.e.:

$$
\begin{aligned}
f a(p) & =\left[\begin{array}{l}
b_{11} \\
b_{21} \\
\cdots \\
b_{K 1}
\end{array}\right] g_{1}(p)+\left[\begin{array}{l}
b_{12} \\
b_{22} \\
\cdots \\
b_{K 2}
\end{array}\right] g_{2}(p)+\ldots+ \\
& +\left[\begin{array}{l}
b_{1 N} \\
b_{2 N} \\
\cdots \\
b_{K N}
\end{array}\right] g_{N}(p)\left[\begin{array}{l}
\bar{a}_{1}^{i} \\
\bar{a}_{2}^{i} \\
\cdots \\
\bar{a}_{k}^{i}
\end{array}\right] .
\end{aligned}
$$

Or written in matrix form:

$$
f a(p)=\left[\begin{array}{cccc}
b_{11} & b_{12} & & b_{1 N} \\
b_{21} & b_{22} & \cdots & b_{2 N} \\
\cdots & \cdots & & \cdots \\
b_{K 1} & b_{K 2} & & b_{K N}
\end{array}\right]\left[\begin{array}{c}
g_{1}(p) \\
g_{2}(p) \\
\cdots \\
g_{N}(p)
\end{array}\right]=B \cdot g(p)
$$

Similar to other examples of Radial Basis Functions (RBF) interpolation, after the basis functions are chosen, we need to solve for the interpolation coefficients collected in matrix $B$.

Having in mind that the values of the function $f a$ to be approximated are collected in the matrix of amplitudes $A$ in the reduced space. This leads to the following equation:

$$
B G=\bar{A} .
$$

Eq. (6) is solved for unknown matrix $B$, and then finally, by combining Eqs. (5) with (3), we arrive to the required general formula for the approximation of the system response for arbitrary parameter combination, which is:

$$
u \approx \bar{\Phi} B g(p)
$$

Eq. (7) was derived by performing the Radial Basis Functions (RBF) interpolation of the system responses in the reduced space, which is represented by amplitude matrix $\bar{A}$ and further transformed by pre-multiplying it by reduced POD basis by [32].

\subsection{Genetic algorithm (GA)}

The Genetic Algorithm (GA) is an evolutionary optimization method, used efficiently for different kinds of optimization problems in last decades [33]. In our study, 10 individuals, also called chromosomes, represent the two damage parameters of position and severity, are converted 
to binary encoding. The population evolves toward better solution iteratively in a process inspired from the natural evolution, where they are allowed to cross among themselves in order to obtain favorable solutions. The fitness is the objective function value, calculated in Eq. (10), as it will be explained latter under section 3 . The best feasible solutions have higher probability to be chosen as parent of new individuals, where the properties of the parents are combined by exchanging chromosomes parts, to produce two new designs. Afterwards, the mutation is performed by randomly replacing the digits of a randomly selected chromosome. These basic operators are repeated to create the next generations until the maximum number of iterations is reached [34].

\subsection{Particle Swarm Optimization (PSO)}

The Particle Swarm Optimization (PSO) is a method inspired by the behavior of different kinds of flocks (birds, bees, fishes, etc.), which is characterized by distinct social and psychological principles. These principles lead the flock to adapt its physical movement towards food seeking in a particular way, which ensures both the speed of the quest and the avoidance of potential adversities, such as hostile predators. This method has been given considerable attention in recent years among the optimization research community.

It is pretty clear that PSO is a population based optimization method built on the premise that social sharing of information among the individuals can provide an evolutionary advantage. The fact that, as an optimization method based on population data, PSO requires a relatively small number of parameters, reduces the computational cost and facilitates the implementation of the algorithm. Due to its simple implementation, PSO can be used in both simple and large-scale optimization problems. Therefore, PSO has been a rather attractive optimization method in scientific circles.

The algorithm was first proposed by Kennedy and Eberhart. PSO has been used widely in the recent years and has been modified in a variety of versions that can handle the majority of optimization problems with or without the presence of constraints.

According to the PSO method, a random population of candidate solutions is considered to be a particle moving through the multi-dimensional design space in search of the position of the global minimum. The particles coexist and cooperate with each other to achieve this position. Every particle can be characterized by its physical position in the design space and its speed of movement. Furthermore, each particle has the ability to remember the best position it has passed so far or personal best (Pbest, Eq. (8)) and the best position that any other particle of the swarm has passed so far or global best (G_best, Eq. (9)).In every iteration the speed of the particle is updated in a stochastic way. Finally, the old and new speed vectors are used in order to update the position of the particle in an iterative manner [35].

The update equations for the speed and the position of the particles are in the following form:

$$
\begin{aligned}
\left\{v^{i}(t+1)\right\}= & w\left\{v^{i}(t)\right\}+c_{1}\left\{r_{1}\right\}\left(\left\{x^{P b, j}\right\}-\left\{x^{j}(t)\right\}\right)+ \\
& +c_{2}\left\{r_{2}\right\}\left(\left\{x^{G b}\right\}-\left\{x^{j}(t)\right\}\right) .
\end{aligned}
$$

$$
\left\{x^{i}(t+1)\right\}=\left\{x^{i}(t)\right\}+v^{i}(t+1) .
$$

\subsection{Description of test structure}

We consider a clamped free beam of pure unidirectional composite of AS4/3501-6 graphite-epoxy materials with symmetrical order of layers. The finite element model is discretized in 10 elements as shown in Fig. 1. Each node of the finite element has three degrees of freedom, normal displacement $w$ along the $z$-axis, rotation $\gamma$ around the $y$-axis and longitudinal displacement $u$ along the $x$-axis. Since the beam is macroscopically considered homogeneous, the shear correction coefficient is the same as for isotropic beam, i.e. $K_{\text {Correction }}=5 / 6$ [36].

The material properties and beam dimensions of AS4/3501-6 graphite epoxy [37] are given in Table 1.

Table 1

Dimension and material of beam

\begin{tabular}{|c|c|}
\hline Ply property & Mean value \\
\hline Length, $\mathrm{m}$ & 0.75 \\
\hline Width, $\mathrm{m}$ & 0.03 \\
\hline Thickness, $\mathrm{m}$ & 0.005 \\
\hline Longitudinal modulus, GPa & 141.96 \\
\hline Transverse Shear modulus, GPa & 6 \\
\hline Density, kg.m-3 & 1600 \\
\hline Poisson's ratio $v$ & 0.42 \\
\hline$K_{\text {Correction }}$ & $5 / 6$ \\
\hline
\end{tabular}

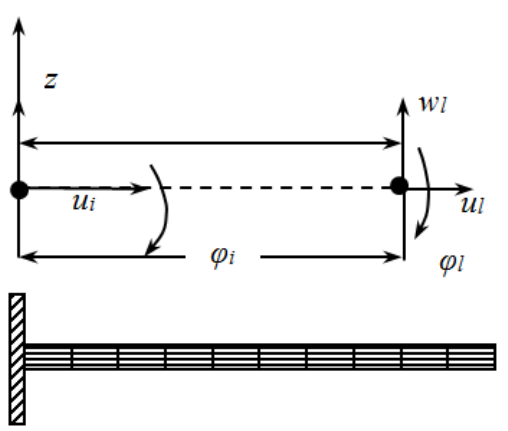

Fig. 1 Beam structure of unidirectionally reinforce graphiteepoxy beam

\section{Objective function}

A practical procedure implemented in a standalone software was developed based on two different optimization algorithms: particle swarm optimization and Genetic Algorithm. The detection and localization task were maintained as an inverse identification problem, where the two parameters of damage position and its level are calculated through the fitness Eq. (10) where the $\omega_{i}^{r}$ is the frequency of the controlled beam, and $\omega_{i}^{c}$ is the frequency calculated using the proper orthogonal decomposition and finite element method:

$$
\text { Fitness }=\sum_{i}^{n}\left(\left(\omega_{i}^{r}-\omega_{i}^{c}\right)^{2} /\left(\omega_{i}^{r}\right)^{2}\right) \text {. }
$$

\subsection{Optimization parameters}

In this study, we address the problem for damage detection and localization in the beam by Genetic Algorithm (GA) and particle swarm optimization (PSO) using FEM and new approach for damage detection and localiza- 
tion using Proper Orthogonal Decomposition (POD).

The particle swarm optimization (PSO) and genetic algorithm (GA) methods were used to minimize the fitness function. In PSO, coordinates of the particles in a two dimensional space are the unknown parameters for damage level and position, using 100 particles. In a second approach, each of the 100 individuals contains two chromosomes representing the required damage parameters. The maximum number of iteration is set equal to 200 .

After several applications, a crossover coefficient of 0.8 and mutation of 0.1 were used in the GA parameters, while $C 1=C 2=2.0$ considered in the PSO method.

\subsection{POD-RBF Based damage detection technique}

During the optimization process, we noticed many approaches that are used to detect and locate damage in beam-like structures using finite element method with optimization techniques. Generally, these techniques require long computation time because each iterative optimization method requires the calculation of location and level of damage. However, our POD approach with RBF can converge in a very short time, while it provides a solution with high accuracy. To build a reduced model by POD-RBF method, 250 FEM results of the studied structure were pro-

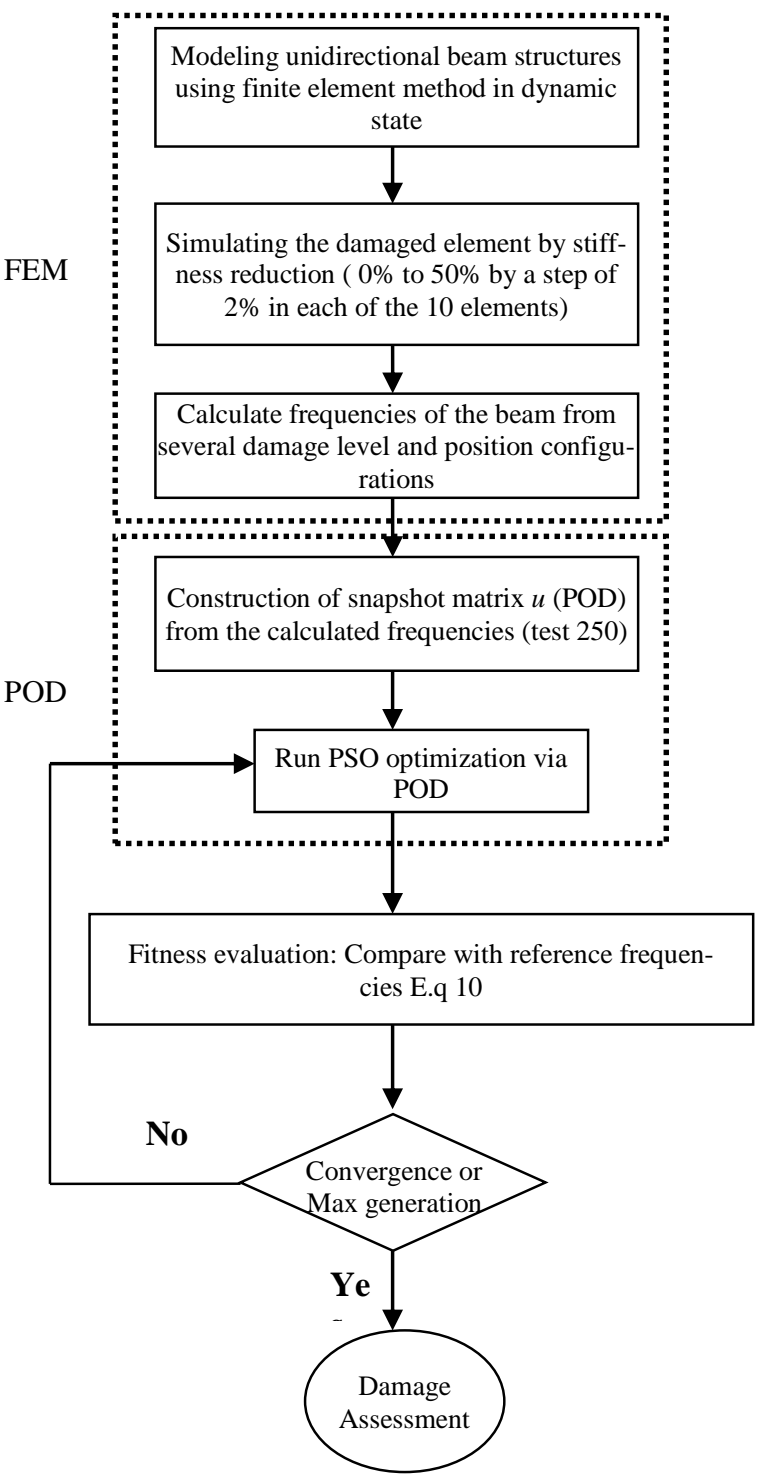

Fig. 2 Methodological approach to the damage detection and localization duced by varying the damage level from $0 \%$ to $50 \%$ using a step of $2 \%$ in each of the 10 elements of the beam. The output parameters considered for the damage identification process are the first five natural frequencies.

The inverse problem is solved using PSO algorithm. The Methodological approach to the damage detection and localization problems is illustrated by a flow chart as shown in Fig. 2.

\section{Results and discussion}

The damage of beam structure was simulated by reducing the stiffness of selected elements by varying amounts using FEM. The largest damage (level 1) consists of $25 \%$ stiffness reduction at the center of damage region followed by $5 \%$ reduction along length of the beam structure. Three different damage locations were studied, namely, near fixed end (D1), center of the plate (D2), and near free edge (D3), as shown in Fig. 3. A modal analysis was performed to determine the natural frequencies of the sane of beam structure and damage cases D1, D2, and D3.

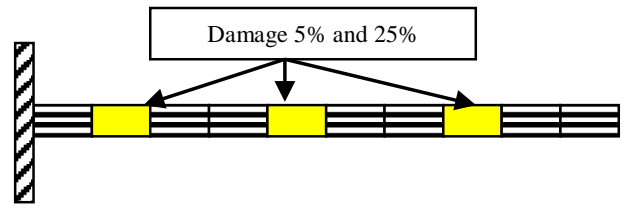

Fig. 3 Damage locations D1 (center), D2 (fixed end), and D3 (free end)

4.1. Damage detection and localization by genetic algorithm and particle swarm optimization using FEM

GA and PSO were used to identify the parameters of the three considered damage scenarios D1, D2 and D3, for damage located at the 2, 5 and 8 elements, respectively with a damage severity of 5 and $25 \%$. The error between real damage and estimated damage by GA and PSO is calculated. A comparison of fitness evolution from three runs of both algorithms is shown in Figs. 4, 5 and 6. The comparison of damage location with GA and PSO are given in the Table 3 .

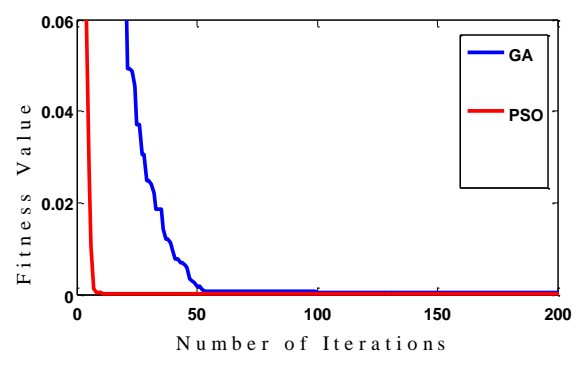

a

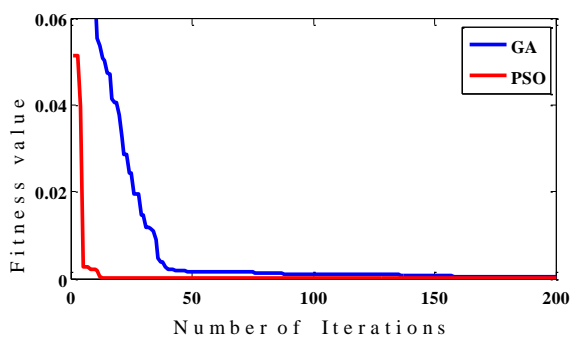

b

Fig. 4 Fitness convergence of GA and PSO using FEM (D1): a - damage 5\%; b - damage 25\% 


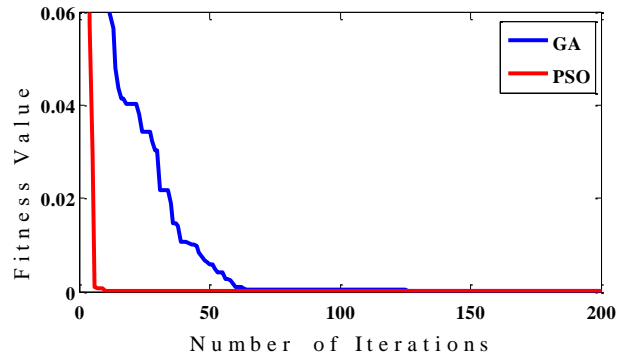

a

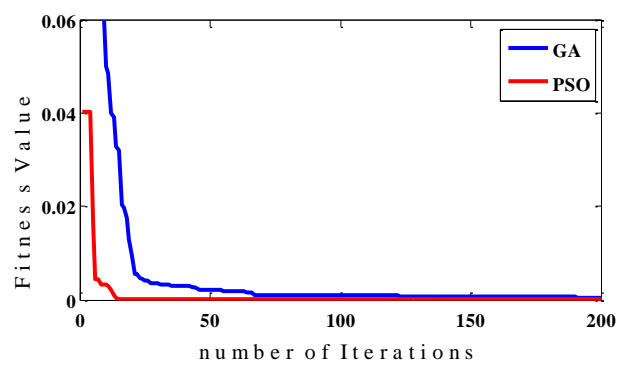

b

Fig. 5 Fitness convergence of GA and PSO using FEM (D2): a - damage 5\%; b - damage $25 \%$

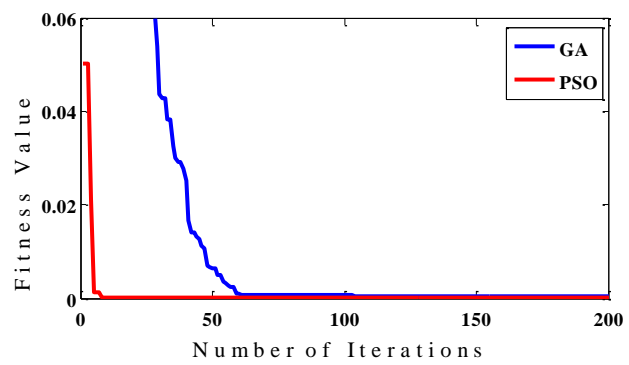

a

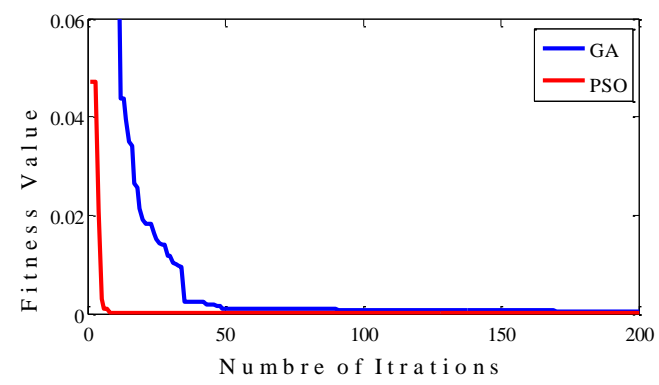

$\mathrm{b}$

Fig. 6 Fitness convergence of GA and PSO using FEM (D1): a - damage 5\%; b - damage $25 \%$

From Figs. 4 to 6 and Table 2, it can be seen that good results are obtained using both PSO and GA algorithms, however PSO is more accurate and faster than GA for the detection and localization of damage. It is noticed that the errors in GA were considerably higher than the errors in PSO. It should be noted that GA, manipulates different mechanisms than PSO. In GA, chromosomes share information with each other so that the whole population moves like one group towards an optimal area. However, the PSO has one way information sharing mechanism, i.e. only $x^{G b}$, which gives out the information to others. The optimization process takes a long time and sometimes the first operation doesn't give the best results, even though several attempts have been made to get the desired results.
Table 2

Comparison between results for damage detection and localization by PSO and GA using FEM

\begin{tabular}{|c|c|c|c|c|c|}
\hline $\begin{array}{c}\text { Damage } \\
\text { element with } \\
\begin{array}{c}\text { Stiffness } \\
\text { reduction (\%) }\end{array}\end{array}$ & Methods & $\begin{array}{c}\text { Damage } \\
\text { element }\end{array}$ & $\begin{array}{c}\text { Stiffness } \\
\text { reduction }\end{array}$ & $\begin{array}{c}\text { Error \% } \\
\text { Damage } \\
\text { element }\end{array}$ & $\begin{array}{c}\text { Error \% } \\
\text { Stiffness } \\
\text { reduction }\end{array}$ \\
\hline D1-2-5 & GA & 2.011 & 4.997 & 0.011 & 0.002 \\
\cline { 2 - 6 } & PSO & 1.999 & 4.998 & 0.001 & 0.002 \\
\hline \multirow{2}{*}{ D2-15-5 } & GA & 4.993 & 4.957 & 0.007 & 0.043 \\
\cline { 2 - 6 } & PSO & 5.004 & 4.999 & 0 & 0.001 \\
\hline \multirow{2}{*}{ D3-8-5 } & GA & 7.998 & 5.000 & 0.002 & 0 \\
\cline { 2 - 6 } & PSO & 7.999 & 5.000 & 0.001 & 0 \\
\hline \multirow{2}{*}{ D1-2-25 } & GA & 2.023 & 24.995 & 0.023 & 0.005 \\
\cline { 2 - 6 } & PSO & 1.999 & 24.998 & 0.001 & 0.002 \\
\hline \multirow{2}{*}{ D2-5-25 } & GA & 4.995 & 24.957 & 0.005 & 0.043 \\
\cline { 2 - 6 } & PSO & 5.000 & 24.999 & 0 & 0.001 \\
\hline \multirow{2}{*}{ D3-8-25 } & GA & 7.991 & 24.9600 & 0.042 & 0.042 \\
\cline { 2 - 6 } & PSO & 5.010 & 25.001 & 0.001 & 0.001 \\
\hline
\end{tabular}

4.2. Damage detection and localization by Particle Swarm Optimization using POD

The proper orthogonal decomposition method (POD) with radial basis function is a well-known model reduction method based on results of the studied phenomenon called the snapshot method. To build a corresponding model of our damaged beam, a snapshot represents a collection of 250 measurements $u$ (see Eq. (1)) of different damage levels [1-25\%] and positions [1-10 element] were considered and the corresponding frequencies are calculated using FEM.

PSO with POD and PSO with FEM were used to identify the parameters of the three considered damage scenarios D1, D2 and D3 located at the 2, 5 and 8 elements, respectively, with a damage severities of 5 and $25 \%$. The error between real damage and estimated damage is calculated. A comparison of fitness evolution for the three damage scenario of both algorithms is shown in Figs. 7 to 9 . The comparison with both approaches is also given in the Table 3.

Table 3 Comparison between results for damage detection and localization by PSO using FEM and POD

\begin{tabular}{|c|c|c|c|c|c|}
\hline $\begin{array}{c}\text { Damage } \\
\text { element } \\
\text { with Stiff- } \\
\text { ness reduc- } \\
\text { tion } \\
(\%)\end{array}$ & $\begin{array}{c}\text { Identification } \\
\text { method }\end{array}$ & $\begin{array}{c}\text { Damage } \\
\text { element }\end{array}$ & $\begin{array}{c}\text { Stiffness } \\
\text { reduction }\end{array}$ & $\begin{array}{c}\text { Error \% } \\
\text { Damage } \\
\text { element }\end{array}$ & $\begin{array}{c}\text { Error \% } \\
\text { Stiffness } \\
\text { reduction }\end{array}$ \\
\hline D1-2-5\% & PSO-FEM & 2.011 & 4.997 & 0.011 & 0.002 \\
\cline { 2 - 6 } & PSO-POD & 2.000 & 5 & 0000 & 0000 \\
\hline D2-5-5\% & PSO-FEM & 4.993 & 4.957 & 0.007 & 0.043 \\
\cline { 2 - 6 } & PSO-POD & 4.9999 & 5.000 & 0.0001 & 0000 \\
\hline D3-8-5\% & PSO-FEM & 7.998 & 5.000 & 0.002 & 0000 \\
\cline { 2 - 6 } & PSO-POD & 8.000 & 5.000 & 0.000 & 0000 \\
\hline D1-2-25\% & PSO-FEM & 2.023 & 24.995 & 0.023 & 0.005 \\
\cline { 2 - 6 } & PSO-POD & 2.000 & 25.0000 & 0.000 & 0000 \\
\hline D2-5-25\% & PSO-FEM & 4.995 & 24.957 & 0.005 & 0.043 \\
\cline { 2 - 6 } & PSO-POD & 5.000 & 24.999 & 0000 & 0.0001 \\
\hline D3-8-25\% & PSO-FEM & 7.991 & 24.9600 & 0.009 & 0.042 \\
\cline { 2 - 6 } & PSO-POD & 8.000 & 25.000 & 0000 & 0000 \\
\hline
\end{tabular}




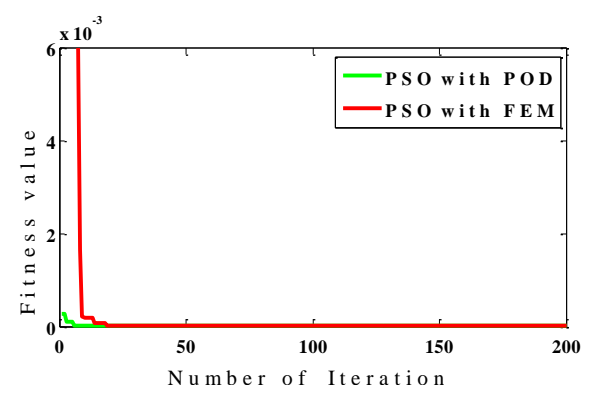

a

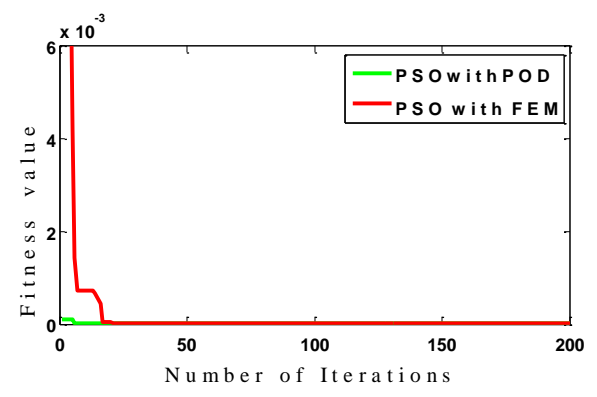

b

Fig. 7 Fitness convergence of PSO using FEM and POD (D1) a - damage $5 \%$; b - damage $25 \%$

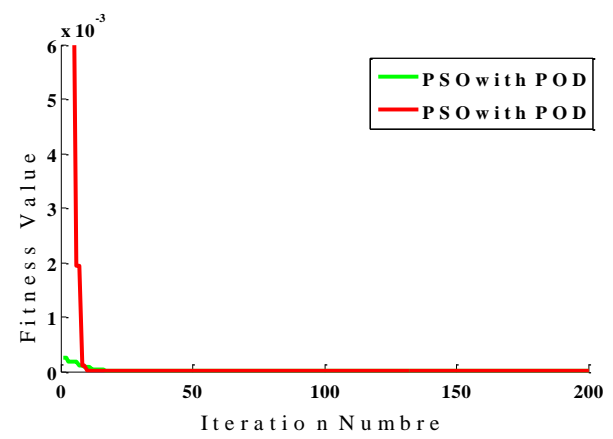

$\mathrm{a}$

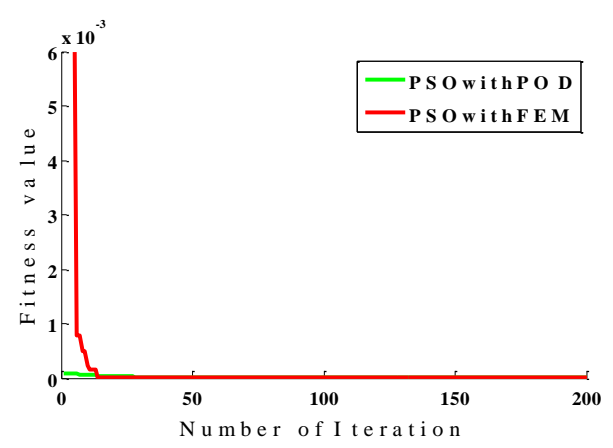

$\mathrm{b}$

Fig. 8 Fitness convergence of PSO using FEM and POD (D2): a - damage $5 \%$; b - damage $25 \%$

From Figs. 7 to 9, it can be seen that the PSO with POD is faster and more accurate than PSO with FEM algorithm to detect and localize damage. The errors are listed in table 3 between real and estimated results for both algorithms. It is noticed that the PSO with POD gives good results with high accuracy and short computation time for locating damage than PSO with FEM. The calculations were carried out using MATLAB program in a PC with characterization Intel(R) core (TM) I3-2328 CPU2.20 GHz. Performing such a test with traditional approach, where system responses are computed by FEM, to solve this current damage detection problem, would require about one hour on an average computer. Moreover, sometimes the desired results may not be obtained. However, using previously calibrated POD-RBF procedure, the results are obtained in a bit more than $50 \mathrm{~s}$ for the first iteration.
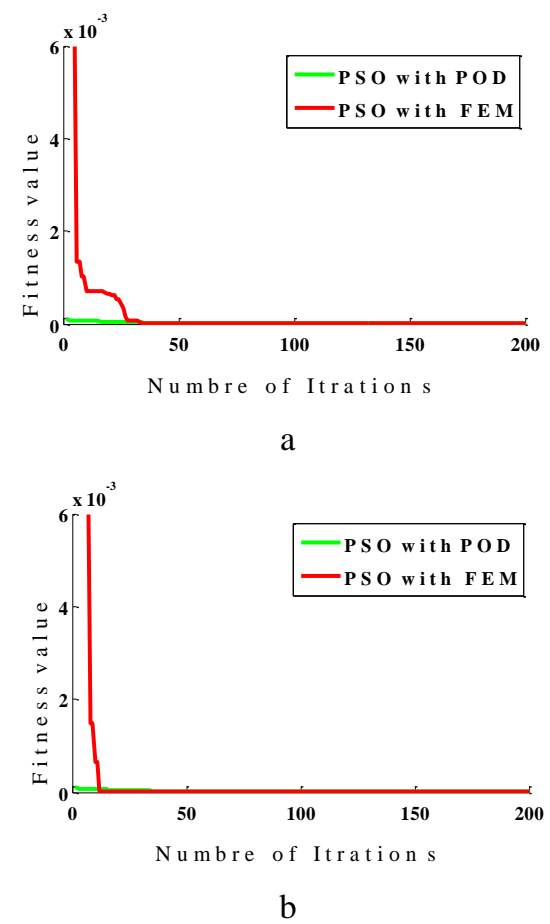

Fig. 9 Fitness convergence of PSO using FEM and POD (D3): a - damage 5\%; b - damage $25 \%$

\section{Effect of noise}

In order to investigate the effect of noise on our damage detection techniques, White Gaussian noise was added to previous results. To test the accuracy of the method using PSO technique, the reference data of the second damage scenario (D2) was manipulated to find natural frequencies, when we consider noise levels of 5\%, $10 \%, 25 \%$ and $50 \%$ as shown in Table 4.

Table 4

Comparison between real damage and estimated damage with noise

\begin{tabular}{|c|c|c|c|c|c|}
\hline $\begin{array}{c}\text { Damage } \\
\text { scenario }\end{array}$ & $\begin{array}{c}\text { Damage } \\
\text { element }\end{array}$ & $\begin{array}{c}\text { Stiffness } \\
\text { reduction } \\
(\%)\end{array}$ & $\begin{array}{c}\text { Noise } \\
(\%)\end{array}$ & $\begin{array}{c}\text { Damage } \\
\text { element } \\
\text { with } \\
\text { noise }\end{array}$ & $\begin{array}{c}\text { Stiffness } \\
\text { reduction } \\
(\%) \text { with } \\
\text { noise }\end{array}$ \\
\hline D2 & 6 & 15 & 1 & 5.99 & 14.987 \\
\hline D2 & 6 & 15 & 5 & 5.981 & 14.971 \\
\hline D2 & 6 & 15 & 10 & 5.974 & 14.968 \\
\hline D2 & 6 & 15 & 15 & 5.970 & 14.962 \\
\hline
\end{tabular}

In Table 4, we compare damage positions and severities, after introducing a perturbation level of $1 \%$, $5 \%, 10 \%$ and $15 \%$. For the cases of $1 \%, 5 \%$, and $10 \%$ noise, we note that there no significant difference in the estimated damage levels. However, at a level of $15 \%$ noise, the difference becomes notable. 


\section{Conclusion}

An approach for detecting and locating damage in beam structures based on model reduction has been investigated at a numerical level using Genetic Algorithm (GA) and particle swarm optimization (PSO) methods to determine damage severities and positions. The results of finite element method (FEM) of the single damaged beam were used to build the snapshot matrix, essential for building a lower order model by the proper orthogonal decomposition. The frequencies of the controlled beam were considered as references in our study.

In the first part of this paper, we run inverse computation using FEM together with PSO and GA, applied to various damage scenarios. The results, in the first part of this study, have shown that PSO using FEM is favorable than GA in damage detection and localization. However, the process takes a considerable amount of time, and requires several iterations to get the desired results. In the second part of this study, we used proper orthogonal decomposition POD with radial basis function RBF to replace FEM in PSO optimization process. The results were found in a very short computing time with high precision compared to FEM-PSO technique. The efficiency of the approach was tested using data with different noise levels.

\section{References}

1. Doebling, S.W.; Farrar, C.R.; Prime, M.B. 1998. A summary review of vibration-based damage identification methods, Shock and Vibration Digest 30: 91-105.

2. Sohn, H.; Farrar, C.R.; Hemez, F.M.; Shunk, D.D.; Stinemates, D.W.; Nadler, B.R.; Czarnecki, J.J. 2004. A review of structural health monitoring literature: 1996-2001, Los Alamos, NM: Los Alamos National Laboratories. Retrieved 2010-07-10.

3. Park, H.; Mackey, R.; James, M.; Zak, M.; Kynard, M.; Sebghati, J.; Greene, W. 2002. Analysis of space shuttle main engine data using beacon-based exception analysis for multi-missions, Aerospace Conference Proceedings, 2002. IEEE. IEEE. http://dx.doi.org/10.1109/AERO.2002.1036123.

4. Adams, R.D.; Coppendale, J. 1976. Measurement of the elastic module of structural adhesives by a resonant bar technique, Journal of Mechanical Engineering Science 18(3): 93-100.

http://dx.doi.org/10.1243/JMES_JOUR_1976_018_025 02.

5. Volkovas, V.; Dulevichus, J. 1975. Identification problems of dynamic models of typical pipe lines parts in diagnostics of the technical state of hydraulic systems (in Russian), Scientific works of higher schools of Lithuania "Vibrotechnika" 24(3): 249-259.

6. Park, H.G.; Zak, M. 2003. Model reconstruction using POD method for gray-box fault detection.

7. Azeez, M.; Vakakis, A. 2001. Proper orthogonal decomposition (POD) of a class of vibroimpact oscillations, Journal of Sound and Vibration 240: 859889.

http://dx.doi.org/10.1006/jsvi.2000.3264.

8. Feeny, B. 2002 On proper orthogonal co-ordinates as indicators of modal activity, Journal of Sound and Vibration 255: 805-817. http://dx.doi.org/10.1006/jsvi.2001.4120.

9. Kerschen, G.; Golinval, J.-C. 2004. Feature extraction using auto-associative neural networks, Smart Materials and Structures 13(1): 211-219. http://dx.doi.org/10.1088/0964-1726/13/1/025.

10. Georgiou, I.T.; Schwartz, I.B. 2001. POD (KL) analysis of the interaction free dynamics of a beam/pendulum configuration: an experimental study, DTIC Document.

11. Kappagantu, R.; Feeny, B. 2000. Part 2: Proper orthogonal modal modeling of a frictionally excited beam, Nonlinear Dynamics 23: 1-11.

12. Volkovas, V.; Klumbys, A.; Ragulskis, K. 1982. Mathematical simulation and vibrodiagnostics of fault states in mechanical systems, "SEECO-82", Environ. Eng. Today. Proc. Pap. Sym. Soc. Environ. Eng., London, pp.7-25.

13. Mozuras, A.; Volkovas, V. 1988. Simulation of defects of beam structure based on flexural vibrations, Vibration Engineering 2(2):75-86.

14. Lanata, F.; Del Grosso, A. 2006. Damage detection and localization for continuous static monitoring of structures using a proper orthogonal decomposition of signals, Smart Materials and Structures 15: 1811-1829.

15. Yazdanpanah1a, O.; Seyedpoor, S.M. 2015. A new damage detection indicator for beams based on mode shape data, Structural Engineering and Mechanics 53(4): 725-744.

http://dx.doi.org/10.12989/sem.2015.53.4.725.

16. Nanda, B.; Maity, D.; Maiti, D.K. 2014. Damage assessment from curvature mode shape using unified particle swarm optimization, Structural Engineering and Mechanics 52(2): 307-322.

http://dx.doi.org/10.12989/sem.2014.52.2.307.

17. Gautier, G.; Serra, R.; Mencik, J.-M. 2012. A subspace fitting method based on finite elements for fast identification of damages in vibrating mechanical systems, Proceedings of ISMA.

18. Cawley, P.; Adams, R.D. 1979. The location of defects instructures from measurements of natural frequencies, Journal of Strain Analysis 14: 49-57.

19. Wang, Y.L. 2014. New damage localization indicator based on curvature for single-span beams, Structural Engineering And Mechanics 51(6): 1037-1046. http://dx.doi.org/10.12989/sem.2014.51.6.1037.

20. Chandra, S.; Barai, S.V. 2014. Damage assessment of frame structure using quadratic time-frequency distributions, Structural Engineering And Mechanics 49(3): 411-425.

http://dx.doi.org/10.12989/sem.2014.49.3.411.

21. Udwadia, F.E. 2005. Structural identification and damage detection from noisy modal data, Journal of Aerospace Engineering 18: 179-187.

22. Lee, Y.-S.; Chung, M.-J. 2000. A study on crack detection using eigenfrequency test data, Computers \& Structures 77: 327-342.

23. Nikolakopoulos, P.; Katsareas, D.; Papadopoulos, C. 1997. Crack identification in frame structures, Computers \& Structures 64: 389-406.

24. Kim, J.-T.; Stubbs, N. 1995. Model-uncertainty impact and damage-detection accuracy in plate girder, Journal of Structural Engineering 121: 1409-1417.

25. Morassi, A. 2001. Identification of a crack in a rod based on changes in a pair of natural frequencies, 
Journal of Sound and Vibration 242: 577-596. http://dx.doi.org/10.1006/jsvi.2000.3380.

26. Choy, F.; Liang, R.; Xu, P.1995. Fault identification of beams on elastic foundation, Computers and Geotechnics 17: 157-176. http://dx.doi.org/10.1016/0266-352X(95)93867-I.

27. Genkin, M.D.; Rusakov, A.M.; Iablonskii, V.V. 1975. Electrodynamic Vibrators, Moscow Izdatel Mashinostroenie, $96 \mathrm{p}$.

28. Kosmatka, J.B.; Ricles, J.M. 1999. Damage detection in structures by modal vibration characterization, Journal of Structural Engineering 125: 1384-1392. http://dx.doi.org/10.1061/(ASCE)07339445(1999)125:12(1384).

29. Hu, N.; Wang, X.; Fukunaga, H.; Yao, Z.; Zhang, H.; Wu, Z. 2001. Damage assessment of structures using modal test data, International Journal of Solids and Structures 38: 3111-3126.

30. Petkevicius, K.; Volkovas, V. 2011. Monitoring and identification of structural damages, Mechanika 17(3): 246-250.

31. Liu, P.-L. 1995 Identification and damage detection of trusses using modal data, Journal of Structural Engineering 121: 599-608. http://dx.doi.org/10.1061/(ASCE)07339445(1995)121:4(599)).

32. Buljak, V. 2011. Inverse Analyses with Model Reduction: Proper Orthogonal Decomposition in Structural Mechanics, Springer. http://dx.doi.org/10.1007/978-3-642-22703-5.

33. Abraham, A.; Jain, L. 2005. Evolutionary multiobjective optimization, Springer London, pp. 1-6.

34. Gen, M.; Cheng, R. 2000. Genetic Algorithms and Engineering Optimization, John Wiley \& Sons, 512 p.

35. Plevris, V.; Batavanis, A.; Papadrakakis, M. 2011. Optimum design of steel structures with the Particle Swarm Optimization method based on EC3, Computational Methods in Structural Dynamics and Earthquake Engineering 2011 (COMPDYN 2011), Corfu, Greece, 25-28 May 2011.

36. Rikards, R. 1999. Analysis of laminated structures, Course of Lectures.
37. Gayathri, P.; Umesh, K.; Ganguli, R. 2010. Effect of matrix cracking and material uncertainty on composite plates, Reliability Engineering \& System Safety 95: 716-728. http://dx.doi.org/10.1016/j.ress.2010.02.004.

Samir Khatir, Idir Belaidi, Roger Serra, Magd Abdel Wahab, Tawfiq Khatir

\section{DAMAGE DETECTION AND LOCALIZATION IN COMPOSITE BEAM STRUCTURES BASED ON VIBRATION ANALYSIS}

S u m m a r y

This paper presents an approach of inverse damage detection and localization based on model reduction. The problem is formulated as an inverse problem where an optimization algorithm is used to minimize the cost function expressed as the normalized difference between a frequency vector of the tested structure and its numerical model. A finite element model of bi-dimensional monolithic composite beam reinforced by a graphite-epoxy is used to define a numerical model of the tested structure in which different scenarios of damage are considered by stiffness reduction. Then, calculations are made on a reduced model built by the technique of proper orthogonal decomposition coupled by radial basis functions. The accuracy of the method is verified through different damage configurations. The results show that the developed algorithm is a feasible methodology of predicting damage in short computing time and with high accuracy. The effect of noise on the accuracy of the results is investigated in some cases for the structure under consideration.

Keywords: Damage detection, localization, beam structure, natural frequencies, eigenvectors, noise, reduced model, and optimization methods.
Received June 18, 2015

Accepted September 17, 2015 